\title{
Retinal vein occlusion: pathophysiology and treatment options
}

This article was published in the following Dove Press journal:

Clinical Ophthalmology

15 July 2010

Number of times this article has been viewed

\author{
Niral Karia \\ Department of Ophthalmology, \\ Southend Hospital, Prittlewell Chase, \\ Westcliff on Sea, Essex, United \\ Kingdom
}

\begin{abstract}
This paper reviews the current thinking about retinal vein occlusion. It gives an overview of its pathophysiology and discusses the evidence behind the various established and emerging treatment paradigms.
\end{abstract}

Keywords: central, hemispheric, branch, retinal vein occlusion, visual loss

\section{Introduction}

Retinal vein occlusion (RVO) is the most common retinal vascular disease after diabetic retinopathy. ${ }^{1}$ Depending on the area of retinal venous drainage effectively occluded it is broadly classified as either central retinal vein occlusion (CRVO), hemispheric retinal vein occlusion (HRVO), or branch retinal vein occlusion (BRVO). Hayreh observed that each of these has two subtypes. ${ }^{2}$ The former two can be subdivided into ischemic and nonischemic CRVO or HRVO, with each having distinct clinical features and prognosis. A number of parameters can be used to assess the degree of ischemia such as the degree of visual loss, presence of a relative afferent pupillary defect, extent of retinal capillary nonperfusion on fluorescein angiography, and electrodiagnostics showing reduced $b$ wave amplitude, reduced $b: a$ ratio and prolonged b-wave implicit time.

BRVO can be considered a major BRVO where a quarter or more of the retina is affected or a macular BRVO where only part of the macular is affected.

Presentation of RVO in general is with variable painless visual loss with any combination of fundal findings consisting of retinal vascular tortuosity, retinal hemorrhages (blot and flame shaped), cotton wool spots, optic disc swelling and macular edema. In a CRVO, retinal hemorrhages will be found in all four quadrants of the fundus, whilst these are restricted to either the superior or inferior fundal hemisphere in a HRVO. In a BRVO, hemorrhages are largely localized to the area drained by the occluded branch retinal vein. Vision loss occurs secondary to macular edema or ischemia.

\section{Epidemiology}

The true incidence of RVO in a population as a whole is difficult to establish, as many RVOs are silent where the condition is mild, the patient is asymptomatic, and it is only detected incidentally. However, longitudinal population based studies have helped in providing an estimate of this incidence. The Blue Mountains Eye Study ${ }^{1}$ found that the 10-year cumulative incidence of RVO was $1.6 \%$ and was significantly associated with increasing age, especially over the age of 70 years. However there was
Correspondence: Niral Karia Department of Ophthalmology, Southend Hospital, Prittlewell Chase, Westcliff on Sea, Essex SSO0RY, United Kingdom Tel +44 I702435 555

Fax +44 I702385877

Email niral.karia@southend.nhs.uk 
no predilection for gender or race. ${ }^{3}$ The Beaver Dam Eye Study $^{4}$ reported a 15-year cumulative incidence of CRVO of $0.5 \%$. For a BRVO this was approximately three times more at $1.8 \%$. Applying this to United Nations projected UK population figures for 2010 gives approximately 47,000 new cases annually. ${ }^{5}$ This figure is greater than 150,000 for the United States. ${ }^{6}$ Rogers et al $^{7}$ carried out a pooled analysis of population based studies from the United States, Europe, Asia, and Australia and projected that approximately 16 million people worldwide may have RVO in at least one eye worldwide. The pooled data showed a higher prevalence of BRVO in Asians and Hispanics compared to whites, although this was not statistically significant, and there was no gender predilection. Whilst less common, it is now generally accepted that (idiopathic) RVO does also occur in the younger (under 50 years) age group, where CRVO tends to be more of the nonischemic type. ${ }^{2}$

\section{Etiology}

Although the exact etiology of RVO remains elusive, it is likely to follow a thrombotic event. In CRVO this may occur in the central retinal vein (CRV) at the lamina cribrosa ${ }^{8}$ or at a variable distance in its journey within the optic nerve posterior to the lamina cribrosa. A more posterior occlusion with a greater number of tributaries of the CRV anterior to the occlusion may allow greater scope for collateral flow to bypass the occluded section of the CRV. ${ }^{2}$ In BRVO, arterial compression of the vein at arteriovenous crossings is thought to incite thrombus formation by causing turbulent flow in combination with pre-existing vascular endothelial damage secondary to systemic cardiovascular risk factors.

In trying to determine etiology or associated risk factors for RVO, comparison is naturally made to factors involved in the occurrence of systemic venous thrombosis (such as deep vein thrombosis). Whilst these two entities may share some common cardiovascular and systemic risk factors, it is also important to understand that they are otherwise quite separate entities requiring different management strategies and leading to different complications. ${ }^{2}$

\section{Systemic vascular/atherosclerotic risk factors in RVO}

Study design, patient characteristics, and risk factor definitions are seldom standardized across the various published papers in the literature. However accounting for this it remains probable that systemic hypertension is the strongest independent risk factor associated with all types of $\mathrm{RVO}^{9-13}$ especially in the older (over 50 years) age group.
Uncontrolled or newly diagnosed hypertension is common in this group, and recurrence of RVO in the same or fellow eye is also noted when hypertension is poorly controlled. In their meta-analysis of 21 studies, O'Mahoney et al ${ }^{12}$ report a significant association between hypertension and both CRVO (pooled odds ratio [OR = 3.8] and BRVO [pooled OR 3.0]. Accepting an inconsistent definition of hyperlipidemia across studies they also found hyperlipidemia to be twice as common in RVO cases (both CRVO and BRVO) compared to controls (pooled OR 2.5). Cheung et $\mathrm{al}^{3}$ also report hypertension and hyperlipidemia as independent risk factors for RVO. The association of diabetes mellitus with RVO is weaker and has not been found to be consistent across all studies. ${ }^{12}$ Its association with CRVO may be stronger than with BRVO. $9,12,13$

\section{Hematological disorders and other systemic conditions}

Conditions that lead to increased blood viscosity such as myeloproliferative disorders are uncommon but known to be associated with CRVO. Similarly, a number of rare systemic inflammatory disorders causing systemic vasculitis (such as Behçet's disease and polyarteritis nodosa) also cause retinal vasculitis leading to RVO, especially in the younger age group. The cause and management of the RVO here is closely linked to the underlying systemic disease and its management.

Over recent years there has been great interest in the potential role of thrombophilia in the development of RVO and in particular CRVO. Thrombophilia refers to the propensity to develop thrombosis (usually venous) due to an abnormality in the coagulation system. This can be congenital (eg, Factor V Leiden, hyperhomocysteinemia or protein $\mathrm{C}$, protein $\mathrm{S}$ and antithrombin deficiencies) or acquired (eg, antiphospholipid syndrome), and its importance is potentially greater in the younger age group. However Fegan's review on CRVO and thrombophilia ${ }^{14}$ suggested that there was a lack of consistency between studies in showing a valid association between CRVO and protein $\mathrm{C}$, protein $\mathrm{S}$ and antithrombin III deficiency, and factor $\mathrm{V}$ Leiden/activated protein $\mathrm{C}$ resistance. These natural anticoagulants are very labile with fluctuating physiological levels. It is recommended that they should be measured on at least two separate samples and if found abnormal confirmed with a third estimation. Most studies used single measurements and varying types of assays. The studies also lacked the statistical power to show a true difference either due to small sample size or lack of a suitable control group. 
In the antiphospholipid syndrome (APS) antibodies to phospholipid activate the coagulation cascade leading to both arterial and venous thrombosis. Tests can be done to either detect the antibody (using the anticardiolipin antibody assay) or its effect on coagulation using a test for lupus anticoagulant. Up to $8 \%$ of patients with APS have ocular manifestations and 4 of 8 studies reviewed by Fegan ${ }^{14}$ showed a significant association of APS in CRVO. Further studies are required to determine the strength of association between APS and RVO.

Homocysteine is a naturally occurring amino acid not found in protein. There are many causes for hyperhomocysteinemia (including rare enzyme deficiencies leading to homocystinuria) which predisposes to both arterial and venous thrombosis. ${ }^{14}$ Several studies have questioned the validity of carrying out exhaustive tests for thrombophilia in RVO in the absence of a suggestive medical history. However their results have shown notable evidence of an association of hyperhomocysteinemia with CRVO sufficient to recommend the benefit of checking for hyperhomocysteinemia, which is correctable with folic acid and vitamins B6 and B12 supplements. ${ }^{14-17}$

On current evidence it would be reasonable to not recommend general thrombophilia screening for all patients with RVO, but to reserve it for older patients with a past history of thromboembolic events and in young patients without any other general risk factors.

\section{Glaucoma/ocular hypertension}

The association between RVO (CRVO in particular) and glaucoma/ocular hypertension has been widely reported d,9,11,13,18 $^{2}$ with the Eye Disease Case-Control Study ${ }^{9}$ reporting an adjusted OR of 5.4 in CRVO for a history of glaucoma. The pathophysiology of this association is unclear, although deformation of the lamina cribrosa in glaucoma may distort the central retinal vein as it exits the eye.

\section{Familial RVO}

Familial clustering of RVO (CRVO in particular) has been reported $^{19,20}$ but these reports have been few in number. It is interesting that such cases are more often bilateral, with a younger age at onset than sporadic cases. More data from existing and future familial clusters is required to establish if there is a genetic cause in these cases.

\section{Pathophysiology of RVO}

It is the occurrence of macular edema in retinal vein occlusion that most frequently leads to visual loss. A working understanding of the pathogenesis of the macular edema may in turn allow an understanding of the mechanism of action of some of the therapies more recently advocated in retinal vein occlusion.

Thrombosis within a retinal vein as described earlier will lead to a partial obstruction of blood flow within the vein and from the eye. The subsequent increased intraluminal pressure, if sufficiently high, will cause transudation of blood products into the retina according to Starling's law. This will result in increased interstitial (retinal) fluid and protein. The latter will increase the interstitial oncotic pressure, perpetuating tissue edema, which will impede capillary perfusion and lead to ischemia. As stated by Campochiaro et $\mathrm{al}^{21}$ this ischemia is not an all or none dichotomy, as those patients classified as nonischemic will still have varying degrees of retinal ischemia.

It is well recognized that inflammation affects the progression and outcome of vitreoretinal disease including retinal vein occlusion. ${ }^{22}$ Yoshimura et $\mathrm{al}^{22}$ have found significantly elevated vitreous levels of the soluble cytokines interleukin (IL) 6 and 8, monocyte chemoattractant protein-1, and vascular endothelial growth factor (VEGF) in RVO, and especially in CRVO. Funk et $\mathrm{al}^{23}$ have also demonstrated elevated aqueous levels of these same factors in patients with CRVO when compared with control samples. The exact interaction of these factors remains speculative but an understanding of the roles that VEGF fulfils is increasing. It is induced by tissue hypoxia such as retinal ischemia and acts as an angiogenic and vasopermeable factor on endothelial cell membrane bound receptors with tyrosine kinase activity. ${ }^{24}$ Ozaki et al ${ }^{25}$ have demonstrated that the implantation of slow release pellets of human recombinant VEGF into the vitreous cavity of rabbits and primates leads to retinal vessel dilatation, breakdown of the blood retinal barrier and retinal new vessel formation. Noma et al have reported elevated aqueous and vitreous levels of VEGF and IL-6 in patients with $\mathrm{BRVO}^{26,27}$ and CRVO, ${ }^{28,29}$ compared to controls. The levels of VEGF and IL-6 correlated with both the severity of macular edema and extent of retinal ischemia (capillary nonperfusion).

It is likely that the sudden retinal ischemia that occurs in BRVO and more so in CRVO will induce excessive VEGF production. VEGF is produced by the retina from retinal pigment epithelial cells, endothelial cells, and Muller cells, as well as other types of ocular tissue. ${ }^{22}$ Boyd et al found a close correlation between aqueous VEGF levels and the course of iris neovascularization and vascular permeability in patients with ischemic CRVO. ${ }^{30}$ The excessive vascular permeability induced by VEGF will likely contribute to the 
macular edema that also occurs according to Starling's law as described above. It is tempting to theorize that even if the primary venous obstruction was overcome (eg, via collateral formation), the macular edema can persist for much longer due to a self perpetuating cycle of VEGF-induced vascular permeability leading to macular edema, capillary damage, and retinal ischemia, stimulating further release of VEGF and other inflammatory cytokines leading to chronic macula edema.

\section{Treatment}

The Branch Retinal Vein Occlusion Study (BRVOS) $)^{31,32}$ and the Central Retinal Vein Occlusion Study (CRVOS) $)^{33,34}$ have established a standard of care by providing both an understanding of the natural history and treatment algorithms for BRVO and CRVO in managing neovascular complications and reducing visual loss. The studies were designed to answer specific questions and so have inherent limitations. Whilst many aspects of these studies may now arguably seem dated, some remain pertinent. In their review of studies evaluating the natural history of CRVO Rogers et $\mathrm{al}^{35}$ confirm that eyes with CRVO had generally poor vision at presentation which declined further with time. They found that over a quarter of nonischemic CRVO converted to ischemic CRVO, of which a quarter developed neovascular glaucoma within 15 months. Similarly they reviewed studies evaluating the natural history of BRVO and reported a general improvement in vision over time without treatment, although improvement beyond 20/40 was uncommon.

\section{Therapeutic options for CRVO}

Mohamed et $\mathrm{al}^{36}$ carried out a systematic review of randomized clinical trials (RCTs) evaluating interventions for the treatment of CRVO. Only results from the CRVOS ${ }^{33,34}$ met the criteria for level 1 evidence. In patients with macular edema secondary to nonischemic CRVO with a vision of $20 / 50$ or worse, macular grid laser photocoagulation does not improve visual acuity although the edema may improve. Additionally prophylactic pan retinal photocoagulation (PRP) in ischemic CRVO does not prevent iris or angle neovascularization and is therefore not recommended. PRP is recommended when anterior segment, disc or retinal neovascularization develop.

Mohamed et $\mathrm{al}^{36}$ also evaluated studies reporting on hemodilution, medical treatment with troxerutin and ticlopidine (inhibitors of platelet aggregation) and intravenous thrombolysis, and various surgical procedures to improve vision in CRVO. By lowering the hematocrit, and thus the plasma viscosity, hemodilution is thought to improve the retinal microcirculation. However the variations in study protocols and the use of multiple agents in combination have prevented any conclusions to be drawn for this treatment modality. Similarly there is limited evidence to recommend the routine use of troxerutin or ticlopidine as well for intravenous thrombolysis, which carries the potential for serious adverse effects such as stroke. The reviews by Squizzato et $\mathrm{al}^{37}$ and Lazo-Langner et $\mathrm{al}^{38}$ suggest that antithrombotic therapy, with low molecular weight heparin (LMWH) in particular, may be efficacious in the treatment of acute RVO with superiority over antiplatelet agents such as aspirin. LMWH appear to have additional properties such as anti-angiogenic effects, which may explain their additional benefits compared to other agents. However the limited evidence available precludes any recommendations about the use of LMWH.

Following a vitrectomy approach, several surgical procedures including internal limiting membrane peel, ${ }^{39}$ radial optic neurotomy, ${ }^{40,41}$ and direct retinal vein cannulation with injection of fibrinolytics, ${ }^{42,43}$ have all been advocated for the management of macular edema in CRVO. However the mechanism of action of these interventions remains contentious and their safety and efficacy have not been evaluated in RCTs. Furthermore carrying out a vitrectomy in itself is thought to improve retinal oxygenation, so confounding the possible effects of the other procedures. Mohamed et al therefore conclude that the routine use of these procedures cannot be recommended.

McAllister et $\mathrm{al}^{44}$ have reported the outcome of the first prospective randomized multicenter trial comparing laserinduced chorioretinal venous anastomosis (L-CRA) with conventional treatment (observation) for CRVO. This technique utilized a high power (argon or Nd:YAG) laser spot to rupture Bruch's membrane and a second spot to rupture a major branch of the retinal vein next to the first laser spot, the intention being to enable an anastomosis to form between the retinal and choroidal circulation. They were able to create a L-CRA in $76.4 \%$ of patients in whom an attempt was made, leading to a significant reduction in the mean retinal fluorescein transit time at 18 months in the treatment group compared to the controls. A mean improvement of 3.6 letters was seen in the treatment group that developed a L-CRA at 18 months compared to a loss of 8.1 letters from baseline in the control group. Although fewer eyes converted to ischemic CRVO in the treatment group compared to controls, $18.2 \%$ of treated eyes developed choroidal neovascularization (CNV) at the treatment site necessitating sector PRP. It remains to be seen whether 
L-CRA becomes widely employed as a treatment option for CRVO. Although the technique is relatively noninvasive and readily accessible it does have a significant learning curve and a high potential rate of complication from CNV.

\section{Therapeutic options for BRVO}

The BRVOS ${ }^{31}$ evaluated whether grid macular laser photocoagulation improved visual acuity (VA) in patients with VA of $20 / 40$ or worse resulting from macular edema secondary to BRVO following at least 3 months of observation. McIntosh et $\mathrm{al}^{45}$ conducted a literature search to identify all relevant RCTs evaluating interventions for BRVO. They concluded that only the results of the BRVOS ${ }^{31}$ met criteria for level 1 evidence - patients treated with grid macular laser gained an average of 1.33 lines at the third year study visit from baseline compared with 0.23 lines in the control group. The grid laser group had statistically significant improvements in VA compared to controls over consecutive visits. Arnarsson and Stefansson ${ }^{46}$ have postulated that destruction of photoreceptors by grid laser leads to increased oxygen flux to the inner retina. An autoregulatory arteriolar constriction and increased resistance then leads to reduced hydrostatic pressure in capillaries and venules, leading to reduced edema with vessel constriction and shortening.

Accepting methodological limitations (such as small sample sizes with insufficient power, short follow up, and lack of a control group), McIntosh et $\mathrm{al}^{45}$ also evaluated studies reporting other interventions including hemodilution, surgery involving pars plana vitrectomy and adventitial sheathotomy, and medical treatment with ticlopidine and troxerutin. They found that these studies lacked sufficient evidence to support the routine use of these other treatment modalities. Muqit et $\mathrm{al}^{47}$ recently reported on the long term vascular perfusion following arteriovenous sheathotomy for BRVO. In their small series they found that long-term epiretinal gliosis and subfoveal photoreceptor atrophy limited the visual recovery.

\section{Intravitreal corticosteroids}

With increasing awareness of the role of VEGF and other inflammatory mediators, the use of off label intravitreal corticosteroids (triamcinolone acetonide in particular) has become routine in the management of RVO in spite of a paucity of RCTs. Small scale studies have reported a positive short/intermediate term efficacy of intravitreal triamcinolone $(\text { IVT })^{48,49}$ but Patel et al ${ }^{50}$ found that whilst IVT was effective in the short term in treating macular edema secondary to all types of RVO, its effectiveness was not maintained after 1 year despite repeated injections.
The exact mechanism of action of corticosteroids in the resolution of macular edema remains speculative. Miyamoto et $\mathrm{l}^{51}$ describe cases where macular edema from RVO or diabetic maculopathy had begun to resolve within 1-6 hours of injecting IVT. They proposed that in addition to the recognized genomic pathway whereby receptor-glucocorticoid interaction is translocated to the nucleus leading to regulation of gene expression and taking many hours or days, there is also a nongenomic pathway. Here the receptor-glucocorticoid complex may act within the cytoplasm to destabilize mRNA, such as VEGF messengers, with rapid effects.

The Standard Care vs Corticosteroid for Retinal Vein Occlusion (SCORE) studies ${ }^{52,53}$ reported RCT data on the efficacy of $1 \mathrm{mg}$ and $4 \mathrm{mg}$ of a preservative free, nondispersive formulation of triamcinolone injected intravitreally. This was compared to the standard of care - observation for macular edema in $\mathrm{CRVO}^{52}$ and grid laser photocoagulation for macular edema in BRVO. ${ }^{53}$ Whilst the SCORE studies have several methodological limitations, as discussed by Apte in his editorial, ${ }^{54}$ they provide important information that modifies the standard of care established by the BRVOS ${ }^{31,32}$ and CRVOS. ${ }^{33,34}$ The SCORE-BRVO study ${ }^{53}$ reported that at the 12 month end point there were no significant differences in visual acuity between the laser treatment, $1 \mathrm{mg}$ and $4 \mathrm{mg}$ groups. The SCORE-CRVO study ${ }^{52}$ however found that subjects in the $1 \mathrm{mg}$ and $4 \mathrm{mg}$ arms were five times more likely to show a gain in visual acuity of 15 letters or more at the 12 month end point compared to observation. Conversely, the study also showed that over three quarters of the eyes that received IVT did not show a gain in vision by 15 letters or more at 12 months and a quarter of treated eyes had a loss of vision of a similar magnitude. The studies also demonstrated a 3-4 times greater rate of intraocular pressure elevation in the IVT (especially 4mg) arms compared to standard of care, and this together with a lack of definitive data to 2 years follow up beckons further studies on IVT and other agents, to search for improved outcomes and better side effect profiles.

Ozurdex (Allergan, Irvine, CA), ${ }^{6}$ a biodegradable intravitreal $700 \mu \mathrm{g}$ dexamethasone implant, received FDA approval in June 2009 for the treatment of macular edema secondary to BRVO or CRVO. Phase III results presented ${ }^{55}$ showed that significantly more patients gained 15 letters or more in the treatment group compared to sham up to 90 days following injection, but this effect waned at 180 days to become nonsignificant. The effects of a repeat injection at 6 months were less pronounced when assessed at 12 months. Although designed to cause less intraocular pressure problems than 
triamcinolone, $25 \%$ of those treated with Ozurdex showed an intraocular pressure rise which peaked at day 60 and returned to baseline by day 180 . The incidence of cataract progression was noted at $4 \%$ in the treatment group, but this increased to $26 \%$ after 1 year where a second injection of Ozurdex had been carried out.

\section{Anti-VEGF treatment}

The anti-VEGF bevacizumab (Avastin, Genetech), a humanized monoclonal antibody binding to all isoforms of VEGF-A, was first reported to show short term efficacy in the resolution of macular edema secondary to CRVO by Rosenfeld in $2005^{56}$ and has since been widely used as an off label treatment in RVO. Prager et $\mathrm{al}^{57}$ have reported a prospective case series of patients with macular edema due to RVO and treated with bevacizumab, showing a mean increase in visual acuity of 16 letters at the 12-month follow up. Subgroup analysis showed a better response in patients with BRVO rather than CRVO, although the reduction in central retinal thickness (CRT) on optical coherence tomography was comparable in both subgroups. This incongruence between functional and anatomical effects was also reported in the SCORE-CRVO study, ${ }^{52}$ where the observation and IVT groups had a comparable reduction in CRT at the 12 month point although visual outcomes were significantly better in the IVT groups.

Ranibizumab (Lucentis; Genentech, San Francisco, CA), approved for the treatment of neovascular age related macular degeneration (n-AMD), is a monoclonal antibody fragment derived from the same parent murine antibody as bevacizumab. The six-month data from two phase III Genentechsponsored studies (BRAVO studying the effects of BRVO and CRUISE studying the effects of CRVO) evaluating the safety and efficacy of Lucentis, compared to sham, for the treatment of macular edema in RVO, were presented at the Retina Congress 2009. ${ }^{58,59}$ BRAVO reported a 7.6 and 7.4 mean letter gain in the $0.3 \mathrm{mg}$ and $0.5 \mathrm{mg}$ study arms of Lucentis respectively, compared to 1.9 letters gained in the sham injection arm. CRUISE reported an 8.8 and 9.3 mean letter gain in the $0.3 \mathrm{mg}$ and $0.5 \mathrm{mg}$ study arms of Lucentis, respectively, compared with 1.1 letters gained in the sham treatment arm. Both studies showed a safety profile consistent with data from previous phase III Lucentis trials for n-AMD. Horizon RVO, an extension trial, will provide much needed longer term data upon completion of BRAVO and CRUISE.

\section{Conclusion}

Studies on n-AMD show that intravitreal treatment is accepted and well tolerated by patients. Corticosteroids and
anti-VEGF medication currently seem to be at the forefront of treatment options for RVO, but RCTs have yet to compare these directly. Corticosteroids can be given as a depot with activity over several months, but the high incidence of intraocular pressure rise and cataract make them less attractive. Intravitreal anti-VEGFs have a low incidence of adverse side effects but are currently short acting requiring frequent injections. Both these agents are used as symptomatic treatments with no defined treatment end points and show high rates of regression and tachyphylaxis with loss of efficacy after repeated injections. There may also be a rebound phenomenon as observed by Matsumoto et al ${ }^{60}$ with macular edema becoming more pronounced compared to pre-treatment levels.

Until a definitive treatment becomes available for RVO it is currently a case of using the various treatment options available to keep the macular dry (to prevent the irreversible damage caused by chronic macular edema) and titrating this to allow a sufficient collateral circulation to develop.

\section{Disclosure}

The author reports no conflicts of interest in this work.

\section{References}

1. Cugati S, Wang JJ, Rochtchina E, et al. Ten-year incidence of retinal vein occlusion in an older population: the Blue Mountains Eye Study. Arch Ophthalmol. 2006;124:726-732.

2. Hayreh S. Prevalent misconceptions about acute retinal vascular occlusive disorders. Prog Retinal Eye Res. 2005;24:493-519.

3. Cheung N, Klein R, Wang J, et al. Traditional and novel cardiovascular risk factors for retinal vein occlusion: the multiethnic study of atherosclerosis. Invest Ophthalmol Vis Sci. 2008;49:4297-4302.

4. Klein R, Moss SE, Meuer SM, Klein BE. The 15-year cumulative incidence of retinal vein occlusion: the Beaver Dam Eye Study. Arch Ophthalmol. 2008;126:513-518.

5. Dexamethasone $700 \mu \mathrm{g}$ intravitreal implant in applicator for retinal vein occlusion. Allergan advance notification document. 2009 Oct.

6. Dexamethasone Intravitreal Implant Approved for Retinal Vein Occlusion. Medscape Medical News. Available from: http://www.medscape. com/viewarticle/704854. Accessed on March 1, 2010.

7. Rogers S, McIntosh RL, Cheung N, et al. The prevalence of retinal vein occlusion: pooled data from population studies from the United States, Europe, Asia, and Australia. Ophthalmology. 2010;117:313-319.

8. Green WR, Chan CC, Hutchins GM, et al. Central retinal vein occlusions: A prospective histopathologic study of 29 eyes in 28 cases. Retina. 1981;1:27-55.

9. The Eye Disease Case-Control Study Group. Risk factors for central retinal vein occlusion. Arch Ophthalmol. 1996;114:545-554.

10. Sperduto RD, Hiller R, Chew E, et al. Risk factors for hemiretinal vein occlusion: comparison with risk factors for central and branch retinal vein occlusion: the eye disease case-control study. Ophthalmology. 1999;106:439-440.

11. The Eye Disease Case-Control Study Group. Risk factors for branch retinal vein occlusion. Am J Ophthalmol. 1993;116:286-296.

12. O'Mahoney P, Wong T, Ray J. Retinal vein occlusion and traditional risk factors for atherosclerosis. Arch Ophthalmol. 2008;126:692-699.

13. Koizumi H, Ferrara D, Brue C, et al. Central retinal vein occlusion case-control study. Am J Ophthalmol. 2007;144:858-863. 
14. Fegan CD. Central retinal vein occlusion and thrombophilia. Eye. 2002;16:98-106

15. Janssen MC, den Heijer M, Cruysberg JR, Wollersheim H, Bredie SJ. Retinal vein occlusion: a form of venous thrombosis or a complication of atherosclerosis? A meta-analysis of thrombophilic factors. Thromb Haemost. 2005;93:1021-1026.

16. Salaun N, Delyfer MN, Rougier MB, Korobelnik JF. Assessment of risk factors for retinal vein occlusions in patients under 60 years of age. $J$ Fr Ophtalmol. 2007;30:918-923.

17. Turello M, Pasca S, Daminato R, et al. Retinal vein occlusion: evaluation of "classic" and "emerging" risk factors and treatment. J Thromb Thrombolysis. 2010;29:459-464.

18. Klein BE, Meuer SM, Knudtson MD, Klein R. The relationship of optic disk cupping to retinal vein occlusion: the Beaver Dam Eye Study. Am J Ophthalmol. 2006;141:859-862.

19. Girmens JF, Scheer S, Heron E, et al. Familial central retinal vein occlusion. Eye. 2008;22:308-310.

20. Bhagat N, Goldberg MF, Gascon W, et al. Central retinal vein occlusion: report of two familial cases. Eur J Ophthalmol. 1999;9:181-195.

21. Campochiaro PA, Hafiz G, Shah SM, et al. Ranibizumab for macular edema due to retinal vein occlusion: implication of VEGF as a critical stimulator. Mol Ther. 2008;16:791-799.

22. Yoshimura T, Sonoda KH, Sugahara M, et al. Comprehensive analysis of inflammatory immune mediators in vitreoretinal diseases. PLOS ONE 2009;4(12):e8158.

23. Funk M, Kriechbaum K, Prager F, et al. Intraocular concentrations of growth factors and cytokines in retinal vein occlusion and the effect of therapy with bevacizumab. Invest Ophthalmol Vis Sci. 2009;50: 1025-1032.

24. Aiello LP, Avery RL, Arrigg PG, et al. Vascular endothelial growth factor in ocular fluid of patients with diabetic retinopathy and other retinal disorders. $N$ Engl J Med. 1994;331:1480-1487.

25. Ozaki H, Hayashi H, Vinores SA, Moromizato Y, Campochiaro PA, Oshima K. Intravitreal sustained release of VEGF causes retinal neovascularization in rabbits and breakdown of the blood-retinal barrier in rabbits and primates. Exp Eye Res. 1997;64:505-517.

26. Noma H, Funatsu H, Yamasaki M, et al. Pathogenesis of macular edema with branch retinal vein occlusion and intraocular levels of vascular endothelial growth factor and interleukin-6. Am J Ophthalmol. 2005;140:256-261.

27. Noma H, Funatsu H, Yamasaki M, et al. Aqueous humour levels of cytokines are correlated to vitreous levels and severity of macular edema in branch retinal vein occlusion. Eye. 2008;22:42-48.

28. Noma H, Funatsu H, Mimura T, Harino S, Sone T, Hori S. Increase of vascular endothelial growth factor and interleukin- 6 in the aqueous humour of patients with macular edema and central retinal vein occlusion. Acta Ophthalmol. Epub 2009 Jun 26.

29. Noma H, Funatsu H, Mimura T, Harino S, Hori S. Vitreous levels of interleukin-6 and vascular endothelial growth factor in macular edema with central retinal vein occlusion. Ophthalmology. 2009;116: 87-93.

30. Boyd SR, Zachary I, Chakravarthy U, et al. Correlation of increased vascular endothelial growth factor with neovascularization and permeability in ischemic central vein occlusion. Arch Ophthalmol. 2002;120:1644-1650.

31. The Branch Vein Occlusion Study Group: Argon laser photocoagulation for macula edema in branch vein occlusion. Am J Ophthalmol. 1984;98:271-282.

32. The Branch Vein Occlusion Study Group: Argon laser scatter photocoagulation for prevention of neovascularization and vitreous hemorrhage in branch vein occlusion. Arch Ophthalmol. 1986;104:34-41.

33. The Central Vein Occlusion Study Group: Evaluation of grid pattern photocoagulation for macular edema in central vein occlusion. Ophthalmology. 1995;102:1425-1433.

34. The Central Vein Occlusion Study Group: A randomized clinical trial of early panretinal photocoagulation for ischemic central vein occlusion. Ophthalmology. 1995;102:1434-1444.
35. Rogers SL, McIntosh RL, Lim L, et al. Natural history of branch retinal vein occlusion: An evidence-based systematic review. Ophthalmology. 2010;117:1094-1101.e5.

36. Mohamed Q, McIntosh RL, Saw SM, Wong TY. Interventions for central retinal vein occlusion: an evidence-based systematic review. Ophthalmology. 2007;114:507-519.

37. Squizzato A, Manfredi E, Bozzato S, et al. Antithrombotic and fibrinolytic drugs for retinal vein occlusion: A systematic review and a call for action. Thromb Haemost. 2010;103:271-276.

38. Lazo-Langner A, Hawel J, Ageno W, Kovacs MJ. Low molecular weight heparin for the treatment of retinal vein occlusion: a systematic review and meta-analysis of randomized trials. Haematologica. 2010; Mar 19 [Epub ahead of print].

39. Mandelcorn MS, Nrusimhadevara RK. Internal limiting membrane peeling for decompression of macular edema in retinal vein occlusion: a report of 14 cases. Retina. 2004;24:348-355.

40. Opremcak EM, Bruce RA, Lomeo MD, et al. Radial optic neurotomy for central retinal vein occlusion: a retrospective pilot study of 11 consecutive cases. Retina. 2001;21:408-415.

41. Weizer JS, Stinnett SS, Fekrat S. Radial optic neurotomy as treatment for central retinal vein occlusion. Am J Ophthalmol. 2003;136:814-819.

42. Weiss JN. Treatment of central retinal vein occlusion by injection of tissue plasminogen activator into a retinal vein. Am J Ophthalmol. 1998; 126:142-144.

43. Bynoe LA, Hutchins RK, Lazarus HS, Friedberg MA. Retinal endovascular surgery for central retinal vein occlusion: initial experience of four surgeons. Retina. 2005;25:625-632.

44. McAllister IL, Gillies M, Smithies LA, et al. The central retinal vein bypass study: A trial of laser-induced chorioretinal venous anastomosis for central retinal vein occlusion. Opthalmology. 2010;117:954-965.

45. McIntosh RL, Mohamed Q, Saw SM, Wong TY. Interventions for branch retinal vein occlusion: an evidence-based systematic review. Ophthalmology. 2007;114:835-854.

46. Arnarsson A, Stefansson E. Laser treatment and the mechanism of edema reduction in branch retinal vein occlusion. Invest Opthalmol Vis Sci. 2000;41:877-879.

47. Muqit MM, Saidkasimova S, Keating D, Murdoch JR. Long-term study of vascular perfusion effects following arteriovenous sheathotomy for branch retinal vein occlusion. Acta Ophthalmol. 2010;88:e57-e65.

48. Park CH, Jaffe GJ, Fekrat S. Intravitreal triamcinolone acetonide in eyes with cystoid macular edema associated with central retinal vein occlusion. Am J Ophthalmol. 2003;136:419-425.

49. Ip MS, Gottlieb JL, Kahana A, et al. Intravitreal triamcinolone for the treatment of macular edema associated with central retinal vein occlusion. Arch Ophthalmol. 2004;122:1131-1136.

50. Patel PJ, Zaheer I, Karia N. Intravitreal triamcinolone acetonide for macular edema owing to retinal vein occlusion. Eye. 2008;22:60-64.

51. Miyamoto N, Iossifov D, Metge F, Behar-Cohen F. Early effects of intravitreal triamcinolone on macular edema. Ophthalmology. 2006;113:2048-2053.

52. The SCORE Study Research Group. A randomized trial comparing the efficacy and safety of intravitreal triamcinolone with observation to treat vision loss associated with macular edema secondary to central retinal vein occlusion: the standard care vs corticosteroid for retinal vein occlusion (SCORE) Study Report 5. Arch Ophthalmol. 2009;127:1101-1114.

53. The SCORE Study Research Group. A randomized trial comparing the efficacy and safety of intravitreal triamcinolone with standard care to treat vision loss associated with macular edema secondary to branch retinal vein occlusion: the standard care vs corticosteroid for retinal vein occlusion (SCORE) Study Report 6. Arch Ophthalmol. 2009;127:1115-1128.

54. Apte RS. SCOREing in retinal venous occlusive disease. Arch Ophthalmol. 2009;127:1203-1204.

55. Loewenstein A, Haller J, Bandello F, et al. 12-month evaluation of an applicator-delivered dexamethasone intravitreal implant in patients with macular edema due to branch or central retinal vein occlusion. Paper presented at: the 33rd Annual Macula Society Meeting; 2010 Feb 26; Tuscon, AZ. 
56. Rosenfeld PJ, Fung AE, Puliafito CA. Optical coherence tomography findings after an intravitreal injection of bevacizumab (avastin) for macular edema from central vein occlusion. Ophthalmic Surg Lasers Imaging. 2005;36:336-339.

57. Prager F, Michels S, Kriechbaum K, et al. Intravitreal bevacizumab (Avastin) for macular edema secondary to retinal vein occlusion: 12-month results of a prospective clinical trial. Br J Ophthalmol. 2009; 93:452-456.

58. Campochiaro PA. Safety and efficacy of intravitreal ranibizumab (Lucentis) in patients with macular edema secondary to branch retinal vein occlusion: The BRAVO Study. Paper presented at: Retina Congress 2009; Oct 4; New York.
59. Brown DM. Safety and efficacy of intravitreal ranibizumab (Lucentis) in patients with macular edema secondary to central retinal vein occlusion: The CRUISE Study. Paper presented at: Retina Congress 2009 Oct 4; New York.

60. Matsumoto Y, Freund KB, Peiretti E, Cooney MJ, Ferrara DC, Yannuzzi LA. Rebound macular edema following bevacizumab (Avastin) therapy for retinal venous occlusive disease. Retina. 2007; 27:426-431.

\section{Publish your work in this journal}

Clinical Ophthalmology is an international, peer-reviewed journal covering all subspecialties within ophthalmology. Key topics include: Optometry; Visual science; Pharmacology and drug therapy in eye diseases; Basic Sciences; Primary and Secondary eye care; Patient Safety and Quality of Care Improvements. This journal is indexed on

\section{Dovepress}

PubMed Central and CAS, and is the official journal of The Society of Clinical Ophthalmology (SCO). The manuscript management system is completely online and includes a very quick and fair peer-review system, which is all easy to use. Visit http://www.dovepress.com/ testimonials.php to read real quotes from published authors. 